\title{
Segmentation of Diabetic Foot Ulcer using Gaussian Mixture Model and Fuzzy KNN Algorithm
}

\author{
C. Karthikeyini, P.Umadevi
}

\begin{abstract}
In recent years image processing gained the attention of many researches on diabetic foot ulcer (DFU) which is caused by strange increase of blood glucose results nerve injury and leads to amputation. This paper discusses about denoising element on wound by GMM method and image segmentation performed using fuzzy K-NN method. The image is denoised by Gaussian mixture model. The fuzzy KNN algorithm is used for segmentation of wound area from the background image. Further, for analysis the segmented area is subjected to homomorphic filtering and gabor filtering. The performance parameters values such as PSNR, MSE and AMBE are calculated from the resultant image. These values are compared with the Gaussian filtered image values.
\end{abstract}

Keywords--- Gaussian Mixture Model, Parameter Measurement, Segmentation on Fuzzy K-NN.

\section{INTRODUCTION}

Diabetic foot ulcer (DFU) is the complication of Diabetic mellitus (DM) where it affects the foot by two ways, such as breaking of neuropathic (nerves) and weakening of vascular (blood vessels). Segmentation is considered as one of the major step which divides a digital image into multiple regions to analyze and distinguish into different objects. The input image is denoised to remove the high frequency noise components. The property of a pixel in an image and information of pixels near to that pixel are two basic parameters for any image segmentation algorithm [1][2]. It can also be representing as similarity of pixels in any region and discontinuity of edges in image. Image segmentation is also used to differentiate different objects in the image, and divided into foreground and background, where foreground of image is related to the region of interest (ROI), and background is the rest of the image [3]. The formation of the wound is generally due to the dead tissue which gets formed at the location where injury occurs, and different techniques are used to capture the image of the wound, for performing the wound assessment. The wound assessment is carried by filtering the wound by removing the air bubbles in order to clean and segment to estimate the healing time required. FCM (Fuzzy C- Means) clustering [4] is used to organize an image into a group which is called as distance based clustering. K-NN is simple algorithm which stores all available cases and classify based on the similarities which is been used in statistical estimation and pattern recognition as a non parametric technique.

Revised Version Manuscript Received on August 19, 2019.

C. Karthikeyini, Senior Professor, Department of Electronics and Communication Engineering, KPR Institute of Engineering and Technology, Coimbatore, Tamil Nadu.

P. Umadevi, PG Scholar, Department of Electronics and Communication Engineering, KPR Institute of Engineering and Technology, Coimbatore, Tamil Nadu.

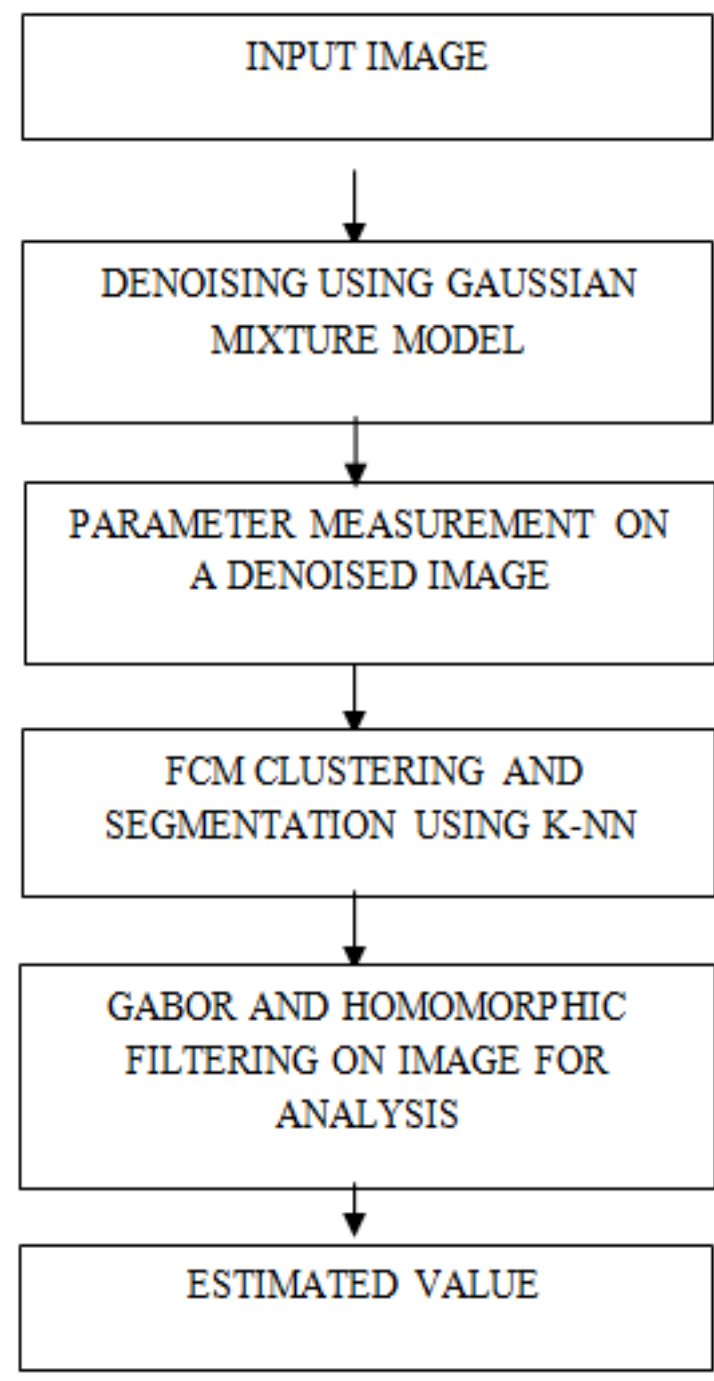

Figure 1.1.1: Block Diagram of Foot Ulcer Technique

\section{GAUSSIAN FILTER MIXTURE MODEL}

Many of the skin segmentation methods can be categorized into the skin color detection approach or the image segmentation approach. The skin color detection approach uses statistical methods for clustering such as histogram and Gaussian mixture model to define the ranges of skin color values in various color spaces. Each cluster is represented by a Gaussian distribution. The clustering process thereby turns to estimating the parameters of the Gaussian mixture, usually by the Expectation-Maximization algorithm [5]. 
In the GMM method, the denoised image is subjected to a low filter analysis to calculate the peak signal to noise ratio (PSNR) between original and filtered image, mean square error (MSE) which is the average of the squared errors between actual and estimated reading, absolute mean brightness error (AMBE) which preserves the original brightness on image are calculated.

$$
\begin{gathered}
M S E=\frac{t 1}{N} ; \\
P S N R=10 * \log 10\left(\frac{255 * 255}{M S E}\right) \\
\text { Where } \mathrm{t} 1 \text { and } \mathrm{t} 2 \text { is given by, } \\
\mathrm{t} 1=\operatorname{sum}\left((\text { clean-denoised }) \wedge^{\wedge}\right) ; \\
\mathrm{t} 2=\operatorname{sum}\left(\text { clean }^{\wedge} 2\right) ;
\end{gathered}
$$

AMBE (absolute mean brightness error),

$$
\begin{gathered}
e m=\operatorname{mean} 2(x) ; \\
m m=\operatorname{mean} 2(m) ; \\
E=\operatorname{abs}(e m-m m)
\end{gathered}
$$

Where,em - expectation mask; $\mathrm{mm}$ - maximization mask

Table 1.2.1: Noise parameters values Estimated by GMM Method

\begin{tabular}{|l|lr|l|}
\hline S.no & \multicolumn{2}{|c|}{ Specification } & Value Estimation \\
\hline 1. & $\begin{array}{l}\text { Peak Signal to Noise } \\
\text { Ratio(PSNR) }\end{array}$ & 11.2759 \\
\hline 2. & $\begin{array}{l}\text { Mean Square Error } \\
\text { (MSE) }\end{array}$ & 4.84 \\
\hline 3. & $\begin{array}{l}\text { Absolute } \\
\text { Brightness }\end{array}$ & $\begin{array}{l}\text { Mean } \\
\text { Error }\end{array}$ & 55.07 \\
\hline
\end{tabular}

(AMBE)

Further the denoised image is been segmented using $\mathrm{K}$ $\mathrm{NN}$ for further analysis. [6].

\section{IMAGE SEGMENTATION USING K-NN METHOD}

The image segmentation approach applies general image segmentation techniques such as clustering, thresholding, edge detection, and region-growing to divide image into different regions based on the discontinuity or homogeneity of the pixel values. In this skin segmentation algorithm is used as a preprocessing step to identify foot ulcer on an input image [7]. For algorithm development and system evaluation, color images of various wound images were collected; two common characteristics were observed from the images collected in the standardized setting. First, pixels of skin representing different parts form homogeneous groups of pixels in the images, and second, skin is usually the largest homogeneous region in the images. Based on these two properties, the Fuzzy C-Means (FCM) algorithm, which is a popular clustering method, was adopted for skin segmentation [8]. The skin segmentation algorithm is illustrated by giving an input RGB image, the image is transformed into the $\mathrm{YCbCr}$ and normalized $\mathrm{RGB}$ color spaces removes the effect of illumination variations and chrominance a colorimetric difference between a given image color and a standard color of equal luminance. It illustrates the clustering on FCM with the normalized values on a predicted wound image.

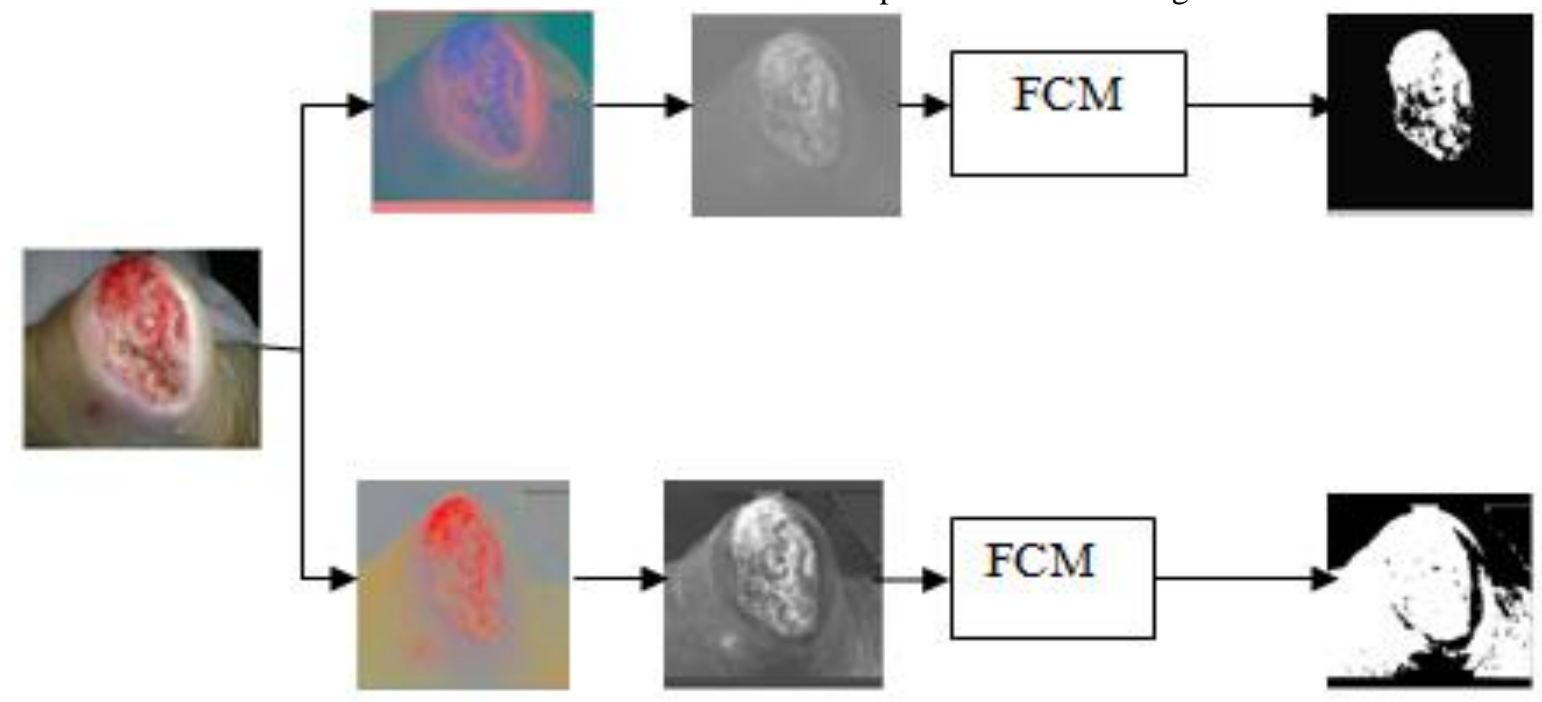

Figure 1.3.1: Illustration of the Fuzzy C-Means (FCM) clustering operation on the $\mathrm{Cr}$ and normalized red channels and the generated skin masks S1 and S2

The normalized RGB color space is defined as

$$
\begin{aligned}
& \text { normR }=R / \sqrt{R^{2}+G^{2}+B^{2}}, \\
& \text { normG }=G / \sqrt{R^{2}+G^{2}+B^{2}}, \\
& \text { normB }=B / \sqrt{R^{2}+G^{2}+B^{2}},
\end{aligned}
$$

Where $\mathrm{R}, \mathrm{G}$, and $\mathrm{B}$ are the intensity values of the red, green, and blue channels respectively.

The $\mathrm{Cr}$ and normalized red channels are then extracted from the corresponding color spaces and smoothed using a mean filter. FCM clustering operation with two clusters is

then applied to the two channels [9]. Since skin tends to have high intensity values in the $\mathrm{Cr}$ and the normalized red channels, the cluster centers are initialized with 10 and 90 percentiles of the intensity values in each channel, where the first cluster represents the background and the second cluster represents the skin. The results given by the FCM clustering operations on the $\mathrm{Cr}$ and the normalized red channels are denoted as skin masks S1 and S2 respectively.

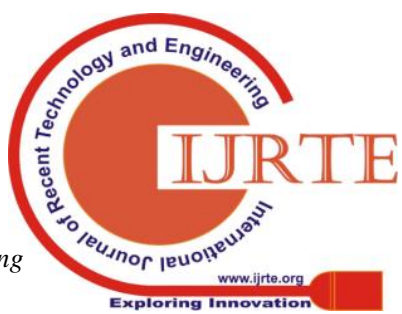


The skin mask S1, which is more accurate at localizing skin color than S2, is then applied to the normalized red channel of the input image to obtain skin-segmented image, where the values of the pixels belonging to the first cluster (background) are set to zero and the values of the pixels belonging to the second cluster (skin) are retained [10].

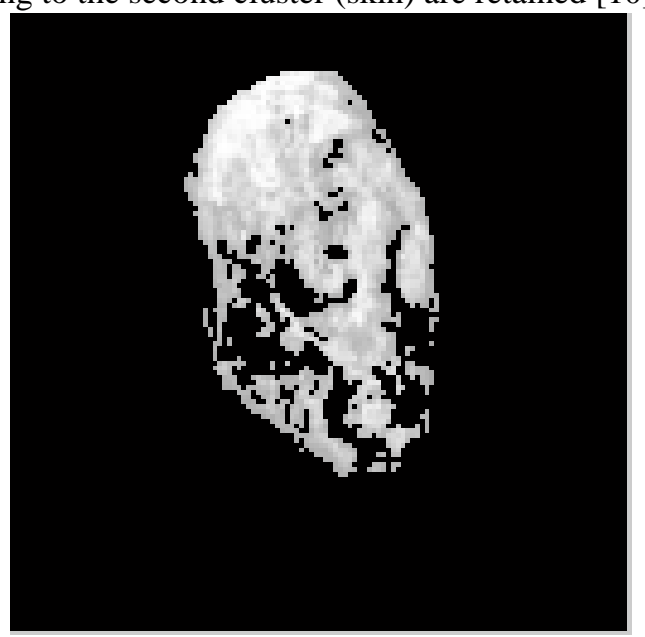

a)Initial segmentation on (S1)

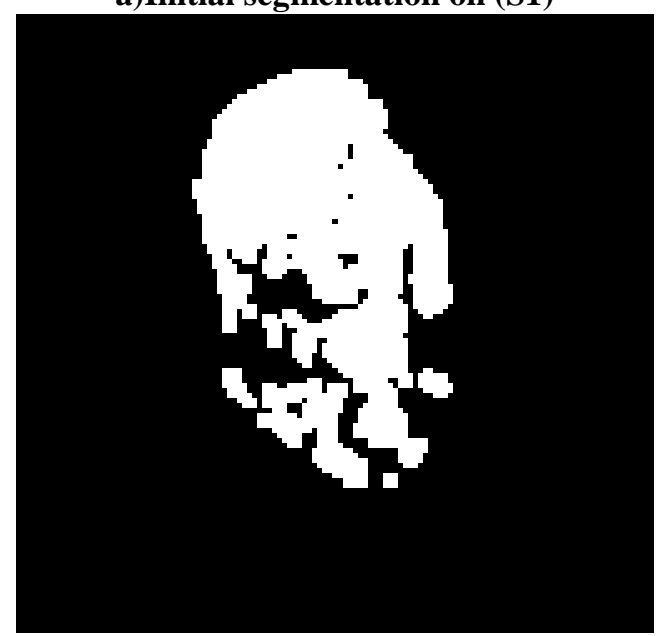

b) Final wound mask

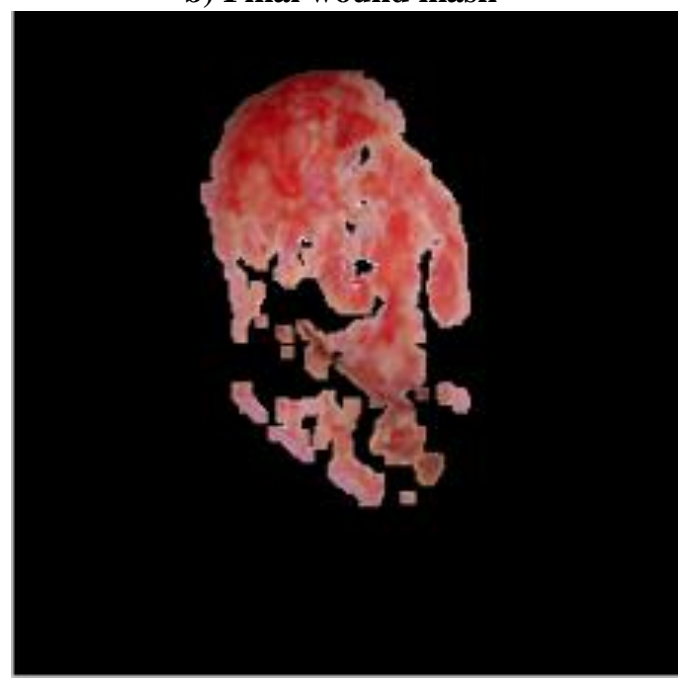

c) Skin Segmentation on color

Figure 1.3.2: a) Initial segmentation on a surrounding skin b) final wounded area analyzed by KNN segmentation c) segmented wound by color feature.

The skin mask S1 is taken for segmentation using K-NN for accurate result a supervised learning technique used in statistical estimation and pattern recognition. The KNN recognized maximum problem on wound and segment the area with the accuracy calculated by,

$$
\text { accuracy }=(t p+t n) / N
$$

Where $\mathrm{tp}=$ true positive; $\mathrm{tn}=$ true negative and $\mathrm{N}=$ number of pixels

$$
\begin{gathered}
t p=\operatorname{sum}(\text { ACTUAL }(\text { idx })==\operatorname{PREDICTED}(i d x)) \\
t n=\operatorname{sum}(\text { ACTUAL }(\sim i d x)==\operatorname{PREDICTED}(\sim i d x))
\end{gathered}
$$

\section{GABOR AND HOMOMORPHIC FILTERING}

After segmentation the filtering is used over for the detection purpose. First the gabor filtering is used a linear filter used for texture analysis which means that it basically analyzes whether there are any specific frequency content in the image in specific direction in a localized region around the point or region of analysis on a segmented wound. Second the homomorphic filtering is a generalized technique for image processing, involving a non linear mapping to a different domain in which linear filter technique are applied for correctness and for brightness error function and image enhancement [11]. Thus image filtering is done over on a blue channel which is sensitive to focus on wound rather than using a red channel.

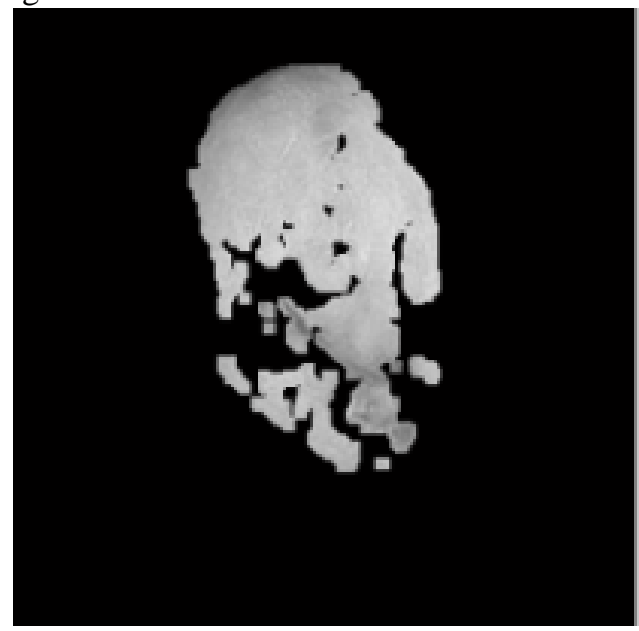

a) Gabor filter on blue Channel

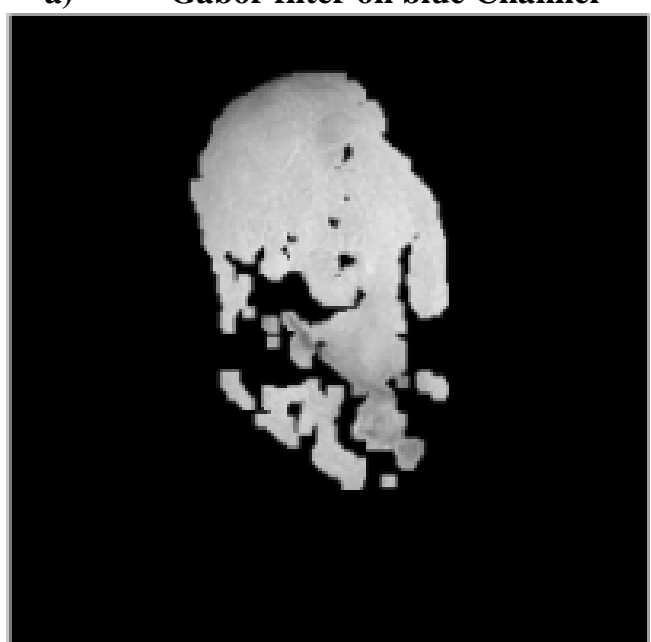

b) Homomorphic filtering

Figure 1.4.1: a) Determines the texture analysis on wound b) Homomorphic filtering for linear operation on brightness detection

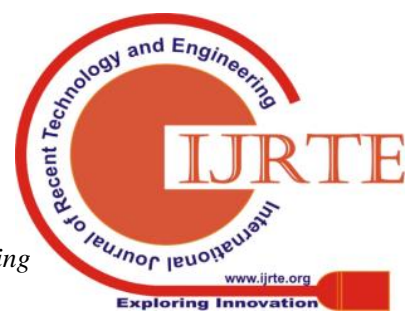


In this process filtering is used to analysis the affected area on the surrounding skin by texture level. Further the analysis on wound states the healing and the non healing level.

\section{RESULT ANALYSIS}

First the image is denoised by GMMand it is segmented by KNN method for analyziation. Thus the mean square error (MSE) and peak signal to noise ratio (PSNR) is measured after the segmentation process and comparison table is figured,

Table 1.5.1: Comparison table on parameter

measurement after the segmentation process

\begin{tabular}{|l|c|c|c|}
\hline S.no & Specification & $\begin{array}{c}\text { Denoised value } \\
\text { /accuracy }\end{array}$ & $\begin{array}{c}\text { Predicted } \\
\text { Value }\end{array}$ \\
\hline 1. & PSNR & 11.27 & 19.48 \\
\hline 2. & $\begin{array}{l}\text { Mean Square Error } \\
\text { (MSE) }\end{array}$ & 4.8 & 2.64 \\
\hline 3. & Accuracy & 89.42 & 98.4 \\
\hline
\end{tabular}

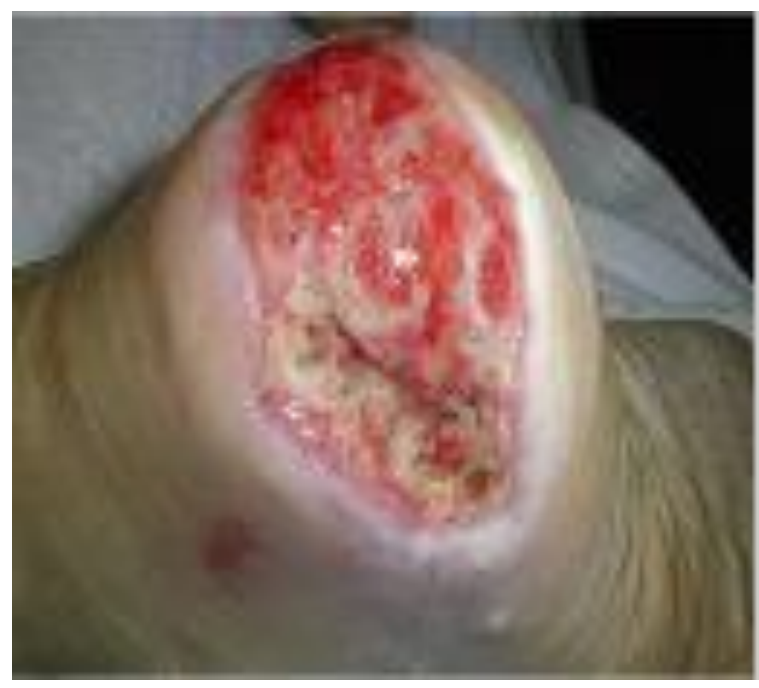

a) Input image

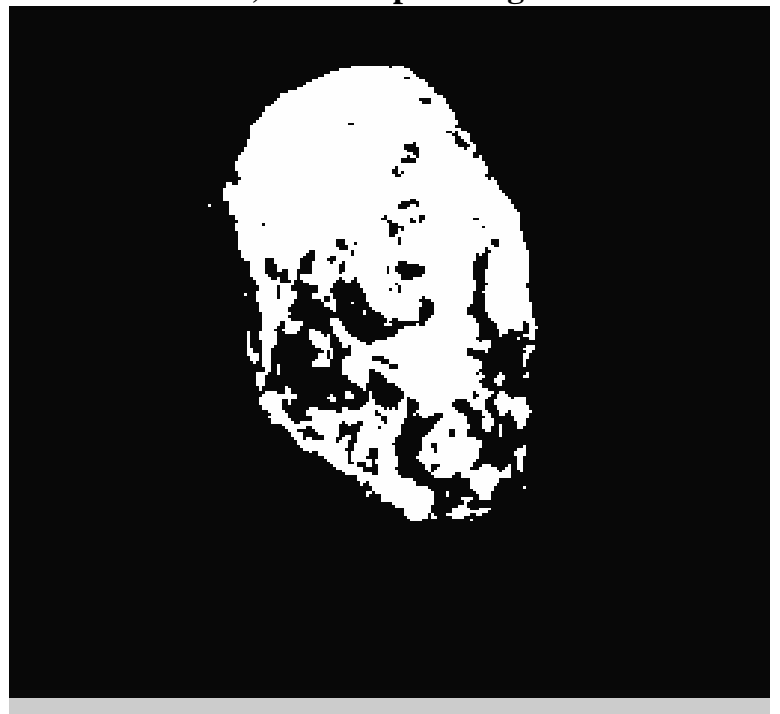

b) GMM denoising

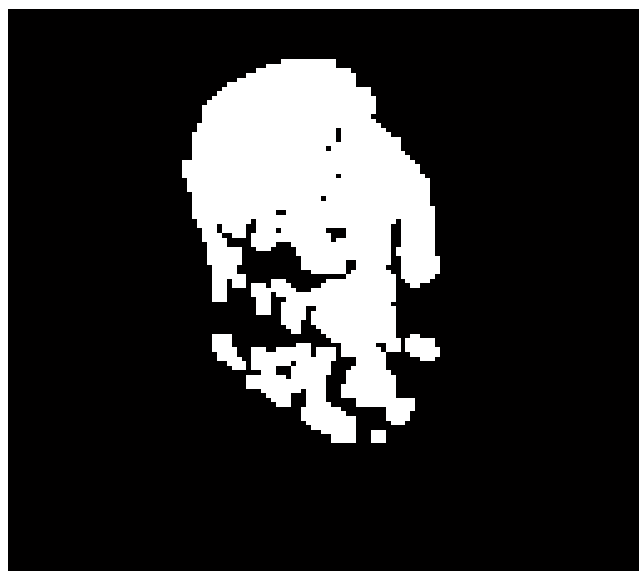

c)Segmentation by KNN

Figure 1.5.2: Result outcome on denoised image and the segmented region on the skin.

\section{CONCLUSION}

A framework for wound segmentation and wound condition analysis, is performed which automatically detect and segment the DFU and surrounding skin area with a high degree of accuracy. These frameworks will be useful for segmenting the other skin lesions such as moles and freckles, spotting marks pimples, other wound pathologies classification, infections like chicken pox or shingles. Thus it is one of the semanticsegmentation that achieves finegrained inference by making dense predictions inferring labels for every pixel, so that each pixel is labeled with the class of its enclosing object or region.

\section{FUTURE WORK}

The proposed system aims at predicting the maximum estimation problem by RPPVSM method (Relatively Permanent Pigmented or Vascular Skin Marks) predicted by non rigid coherent point drift method [12]. RPPVSM includes two section namely i.e.) RPPVSM detection and matching, where in this RPPVSM detection method is performed for detecting deep wounded area and matching is done where the prediction pixel status the healing and non healing process [13][14].

\section{REFERENCES}

1. W. J. Jeffcoate and K. G. Harding, "Diabetic foot ulcers," The lancet, vol. 361, no. 9368, pp. 1545-1551, 2003

2. N.R. Pal and S.K. Pal, "A Review on Image Segmentation Techniques,” Pattern Recognition, vol. 26, no. 9, pp. 1277-1294, 1993.

3. R.M. Haralick and L.G. Shapiro, "Image Segmentation Techniques," Computer Vision, Graphics, and Image Processing, vol. 29, no. 1, pp. 100-132, 1985.

4. A. Castro, C. Boveda, and B. Arcay, "Analysis of fuzzy clustering " algorithms for the segmentation of burn wounds photographs," in International Conference Image Analysis and Recognition. Springer, 2006, pp. 491-501.

5. Armand Moulis "Automatic Detection and Classification of Permanent and Non-Permanent Skin Marks Using Gaussian Mixture Model".

6. H.D. Cheng, X.H. Jiang, Y. Sun, and J. Wang, "Color Image Segmentation: Advances and Prospects," Pattern Recognition, vol. 34, no. 12, pp. 2259-2281, 2001.

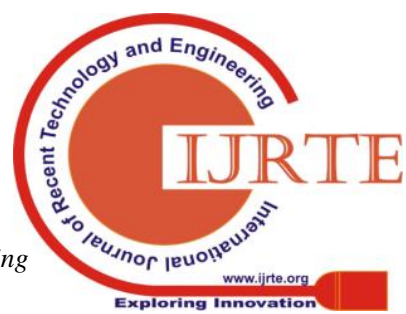


7. P. Kakumanu, S. Makrogiannis, and N. Bourbakis, "A Survey of Skin-Color Modeling and Detection Methods," Pattern Recognition, vol. 40, no. 3, pp. 1106-1122, 2007.

8. V. Vezhnevets, V. Sazonov, and A. Andreeva, "A Survey on PixelBased Skin Color Detection Techniques," in Proc. Graphicon, pp. 85 92, 2003.

9. P. Kakumanu, S. Makrogiannis, and N. Bourbakis, "A Survey of Skin-Color Modeling and Detection Methods," Pattern Recognition, vol. 40, no. 3, pp. 1106-1122, 2007.

10. J.C. Bezdek, Pattern Recognition with Fuzzy Objective Function Algorithms, New York: Plenum Press, 1981.

11. AndriyMyronenko, Xubo Song Miguel A, Carreira-Perpin "Non-rigid point set registration: Coherent Point Drift".

12. A. Nurhudatiana, A.W.K. Kong, L. Altieri, and N. Craft, "Automated Identification of Relatively Permanent Pigmented or Vascular Skin Marks (RPPVSM)," in Proc. ICASSP, pp. 2984-2988, 2013.

13. C. Tang, A.W.K. Kong, and N. Craft, "Uncovering Vein Patterns from Color Skin Images for Forensic Analysis," in Proc. CVPR, pp. 665-672, 2011.

14. H. Zhang, C. Tang, A. Kong, and N. Craft, "Matching Vein Patterns from Color Images for Forensic Investigation," in Proc. BTAS, pp. 77-84, 2012 .

15. http://www.imageprocessing place.com//

16. http://github.com/giannisnik/cnn.graph-classification. 